\title{
The Role of Artificial Intelligence in the Prediction of Functional Maturation of Arteriovenous Fistula
}

\author{
Ali Kordzadeh, MBBS, MSc, MD, VA-BC, FEBS, FEBVS1,3 and Shabnam Sadeghi Esfahlani, BSc, PhD²
}

Objective: The aim of this study is to examine the application of virtual artificial intelligence (Al) in the prediction of functional maturation (FM) and pattern recognition of factors in autogenous radiocephalic arteriovenous fistula (RCAVF) formation.

Materials and Methods: A prospective database of 266 individuals over a four-year period with $n=10$ variables were used to train, validate and test an artificial neural network (ANN). The ANN was constructed to create a predictive model and evaluate the impact of variables on the endpoint of FM.

Results: The overall accuracy of the training, validation, testing and all data on each output matrix at detecting FM was $86.4 \%, 82.5 \%, 77.5 \%$ and $84.5 \%$, respectively. The results corresponded with their area under the curve for each output matrix at best sensitivity and at 1-specificity with the log-rank test $p<0.01$. ANN classification identified age, artery and vein diameter to influence FM with an accuracy of $(>89 \%)$. Al has the ability of predicting with a high grade of accuracy FM and recognising patterns that influence it.

Conclusion: $\mathrm{Al}$ is a replicable tool that could remain up to date and flexible to ongoing deep learning with further

${ }^{1}$ Faculty of Medical Sciences, Anglia Ruskin University, Cambridge, $U K$

${ }^{2}$ Faculty of Science and Technology, Anglia Ruskin University, Cambridge, UK

${ }^{3}$ Department of Vascular, Endovascular and Renal Access Surgery, Broomfield Hospital, Mid Essex Hospital Service NHS Trust, Essex, UK

Received: October 22, 2018; Accepted: January 8, 2019 Corresponding author: Ali Kordzadeh, MBBS, MSc, MD, VABC, FEBS, FEBVS. Department of Vascular, Endovascular and Renal Access Surgery, Broomfield Hospital, Mid Essex Hospital Service NHS Trust, Court Road, Chelmsford, Essex CM1 7ET, UK

Tel: +44-1245-513497, Fax: +44-1245-515222

E-mail: alikordzadeh@gmail.com

(cc) BY-NC-SA (C2019 The Editorial Committee of Annals of Vascular Diseases. This article is distributed under the terms of the Creative Commons Attribution License, which permits use, distribution, and reproduction in any medium, provided the credit of the original work, a link to the license, and indication of any change are properly given, and the original work is not used for commercial purposes. Remixed or transformed contributions must be distributed under the same license as the original. data feed ensuring substantial enhancement in its accuracy. Al could serve as a clinical decision-making tool and its application in vascular access requires further evaluation.

Keywords: artificial intelligence (Al), artificial neural network (ANN), radiocephalic arteriovenous fistula (RCAVF), functional maturation (FM), pattern recognition

\section{Introduction}

Radiocephalic arteriovenous fistula (RCAVF) is the primary choice of vascular access for haemodialysis patients. ${ }^{1)}$ Upon functional maturation (FM) the RCAVFs remain patent for a few years and demand limited interventions. However, FM varies from one unit to another in the range from $40 \%-70 \%$. 2) The impact of multiple variables on the FM of RCAVF has been extensively investigated, and to date it has become apparent that certain variables might impact FM. ${ }^{3)}$ However, conflict regarding their independent impact, association and causal link is persistent, and despite conclusive evidence of a few series, this is negated by others and recent guidelines remain inconclusive. ${ }^{4)}$ This has been attributed to the fact that the majority of such outcomes are derived from cohort studies, and despite all efforts they could be subjected to selection, performance and conclusion bias or could lack randomisation. ${ }^{4)}$ Furthermore, the type of statistical analysis deployed by various investigators is varied and not unified, and according to the words of Mark Twain: 'facts are stubborn things, but statistics are pliable.'5) This creates a patient stratification dilemma for units, and to date different scoring systems have simply failed to aid clinicians in detecting the best candidates for RCAVF formation or for prediction of their outcome. Even if they do, they are not replicable in other centres. ${ }^{6,7)}$

Artificial intelligence (AI), a branch of computer science, has two subtypes of virtual and physical application in the field of medicine. Physical application of AI is well established and reflected in the use of the 'Da Vinci' surgical robot utilised in the treatment of various gynaeco- 
logical, urological and colorectal conditions. ${ }^{8)}$ The virtual component or so-called 'machine learning' is applicable to almost every discipline of medicine that conducts multifactorial analysis ranging from descriptive to predictive analytics. Their potential to utilise an important link within a data set can be used in the diagnosis, treatment and in prediction of outcomes in various clinical circumstances. However, their application in vascular surgery and access provision remains limited and unexplored. ${ }^{9)}$ A major advantage of $\mathrm{AI}$ is related to its ability to negate all setbacks that are associated with biased biostatistics, study design and conclusion by eliminating the human error. Furthermore, an ongoing and continuous data feed in AI could eventually produce a deep learning system that could independently predict, detect and evaluate longterm trajectories that are associated with outcomes in any field of surgery. $\left.{ }^{8}{ }^{91}\right)$ Therefore, the primary objective of this study is to assess the ability of virtual AI (artificial neural network [ANN]) for the first time in literature to predict FM of RCAVF. The secondary aim is to evaluate factors that might impact FM.

\section{Methods}

A prospective consecutive cohort study on $n=266$ patients who underwent RCAVF from 1 May 2012 to 1 May 2016 was conducted. Data on patient's demographics (age, gender), anatomical variance (cephalic vein \& radial artery diameter), comorbidities (diabetes mellitus [DM], ischaemic heart disease [IHD], congestive heart failure $[\mathrm{CHF}]$, hypertension [HTN]), type of anaesthesia (local vs. general) and presence of pulsation, bruit and thrill was obtained. Data was completely anonymised and was fed into ANNs. Access to local renal data registry was granted through audit number CA13-225. The study was conducted in accordance with the Helsinki Code of ethical principles and no patient was subjected to any new, alternative or change of practice.

\section{Standards and definitions}

The outcome of the study was set on the endpoint of FM. FM was defined following the 'Rule of 6's' assessed clinically and with duplex ultrasonography at six weeks post RCAVF formation. ${ }^{10)}$ This included depth of not more than 0.5 to $0.6 \mathrm{~cm}$ from skin, and diameter (main body of fistula) of $6 \mathrm{~mm}$ with a flow rate of $600 \mathrm{ml} / \mathrm{min}$ and a length of 5 to $6 \mathrm{~cm}$ for successful two-needle cannulation and haemodialysis. ${ }^{10)}$ RCAVFs that did not achieve FM by six weeks were categorised as FM failure. Follow-up was set at one, four and six weeks from the time of creation depending on the status of the fistulas. All fistulas were created by senior vascular access providers and were created on the non-dominant hand with 'fistula first initiative.'
Preoperative Doppler Ultrasound (DUS) was used to assess cephalic vein (non-tourniquet) diameter (internal calibre) and its direct or indirect continuation to the median cubitan fossa. This calculation involved measurement of the vein diameter at three different fixed sites, resulting in a mean value which is represented in this manuscript. In addition, radial artery (haemodynamic studies) was conducted using DUS, and no patient in this cohort had any inflow issues or was subjected to inflow angioplasty. All comorbidities were categorised and defined in accordance with definitions provided by the World Health Organisation. ${ }^{11)}$ All cases of congestive cardiac failure (CCF) classified according to the American Heart Association were in level A, and no patient with CCF category B or C had an AVF formation. ${ }^{12)}$ This is because presence of level B or C (CCF) will worsen with AVF formation, contributing to mortality and morbidity, and this should be limited in practice.

\section{ANNs}

ANN is a brained-inspired system (neurons and synapses) that is intended to replicate the human brain by learning. When the data gets too complex or large, they are able to recognise patterns and predict outcomes based on logistic and sigmoid units that are adapted for real-time diagnosis and classification. ${ }^{13)}$

ANN consists of layers of neurons that constitute an input layer (given data), one or more middle or hidden layers (analysis/perception/detection section) and an output layer (outcome). The neurons are connected by links, and each link has a numerical weight associated and assigned to it. To perform various sets of analysis on the given data (input), the signals travel from the first layer (input) to the last one (output) only if the aggregate signal passes the required limit. ANN learns through repeated adjustments of these weights. The predictive model is a threestage weighted neural learning network with 10-input, 10-hidden and 2-output layers. The output has two elements of positive (FM) and adverse outcome (failure of FM). The final result is an algorithm, which is based on independent training and machine learning.

One of the most vital characteristics of ANNs is that they can learn from their experience in a training environment. The use of multilayer feedforward perceptron is restricted by the lack of a suitable learning algorithm with integrated backpropagation learning. This simply implies that data is analysed in various ways so that it can represent the outcome if tested again, and if there is misrepresentation, this is fed backwards (backpropagation) until an ideal representation (pattern recognition) is achieved before final output layer. The learning process continues as long as the network improves its performance on endpoints of validation and accuracy by crossing the input 


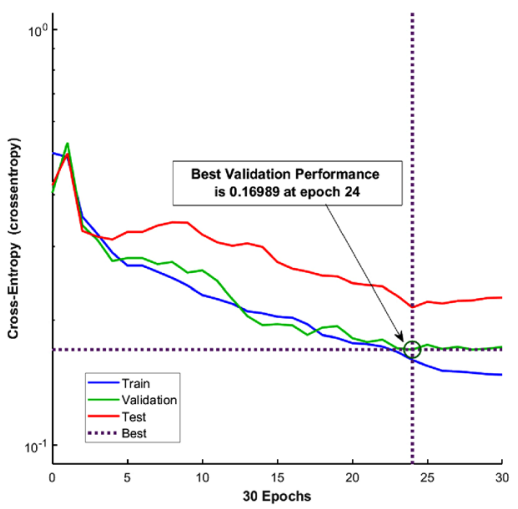

(a)

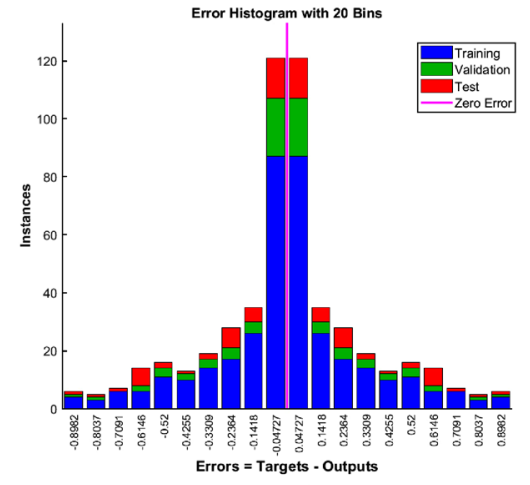

(b)

Fig. 1 Artificial neural network training performance.

(a) The training stopped once the mean squared error for validation data reached its minimum value (at epoch 24); (b) a bell-shaped (normal distributed) histogram for error (target-output) of instances.

layers multiple times to train the ANN. ${ }^{14)}$ For optimisation of parameters, the data is automatically divided into three sections of $70 \%$ for training, $15 \%$ for evaluation and the F-measure (combination of precision and recall) and $15 \%$ for validation. Once training on $70 \%$ of the data is completed, the predictive model is evaluated and measured for its independent precision and for recall with final validation on the remaining $15 \%$. This is conducted automatically without human interference and the testing data set does not affect training and provides an independent measure of network performance during and after training. Overall, ANN could be compared to a neuron (nerve cell) where information is directed towards the cell body (input data [node]) and then transmitted through the axon (input repetition [node] and weighting) and later to the synapses (output [node]) only in a mathematical format (computer) with a numerical outcome rather than an electrical impulse.

\section{Analysis}

This machine learning algorithm included the following unbiased/unweighted information: Age at the time of surgery (numeric); presence of thrill/pulse $(0=$ no thrill or pulse, $1=$ thrill or pulse) (binary), gender ( $0=$ female, $1=$ male) (binary); DM (binary); IHD (binary); CHF (binary); HTN (binary); vein size in millimetres $(\mathrm{mm})(\mathrm{nu}-$ meric); and artery size in millimetres ( $\mathrm{mm}$ ) (numeric). The matrix defining 10 attributes of 266 subjects $(10 \times 266)$ and the target was set on two characteristics of a 266 $(2 \times 266)$ matrix, where each column indicates a correct category and is performed in MATLAB and Statistics Toolbox (Release 2017, The MathWorks, Inc., Natick, MA, USA). More variables (attributes) could not be included as the complete dataset was only available on the given $\mathrm{n}=266$ with 10 attributes. The initial stage involves the formation of scatter plots in 2 dimensional (2D) and
3 dimensional (3D) with the range of data (variables) and their accumulation around a point. The data is ultimately assessed through backpropagation which is the method of calculating the 'gradient' required for weights of each variable for ANN. The data areas eventually iterated over one epoch (reference point on a scale from which time is measured), and this is validated at a range of 0-6 (six being the highest level of validation) on a scale. The probability distribution of the ANN performance on training and validation data is assessed through cross entropy along with error measurement (histogram). The training and the network were adjusted according to its error, and the validation sample was used to measure network generalisation and to halt training when generalisation stopped improving. Finally, an individual matrix for training, validation, testing and overall outcome for ANN was conducted, and the findings of each matrix were validated through the corresponding area under the curve (AUC) for best sensitivity and 1-specificity in percentage with the log-rank test.

\section{Results}

FM was obtained in $67 \%(n=178 / 266)$ of the cohort. The mean age of the cohort was 63 years of age (interquartile range [IQR], 22-88). There was prevalence of a $3: 1$ male to female $(75.5 \%, n=201 / 266$ vs. $24.5 \%$, $\mathrm{n}=65 / 266$ ). Local anaesthesia was the anaesthetic of choice in $94 \%$ of the cohort $(n=250 / 266$ vs. $n=16 / 266$ general anaesthesia). The most common comorbidity was HTN at $81 \%(\mathrm{n}=216 / 266)$, followed by DM at $35.7 \%$ $(\mathrm{n}=95 / 266)$, IHD at $21 \%(\mathrm{n}=58 / 266)$ and CCF type A at $4.1 \%(\mathrm{n}=11 / 266)$. The non-augmented (no-tourniquet) vein diameter was $1.7 \mathrm{~mm}$ (IQR, 0.8-3.5) and this value for radial artery was $1.9 \mathrm{~mm}$ (IQR, 1-3.4).

The training of the ANN continued until it reached the 


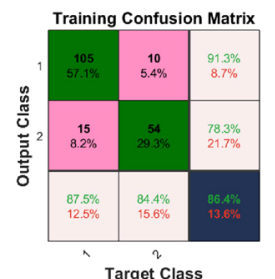

(a)

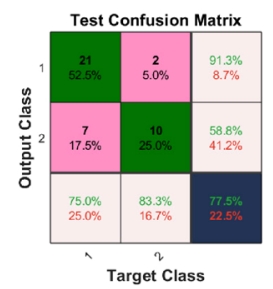

(c)

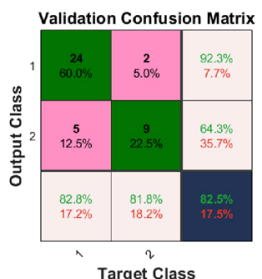

(b)

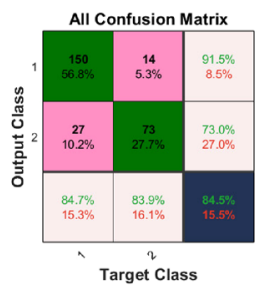

(d)

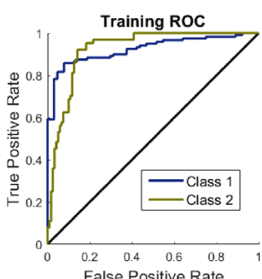

(e)

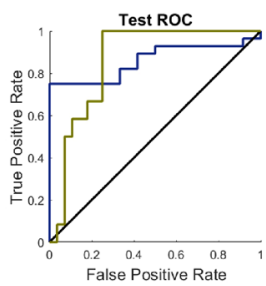

(g)

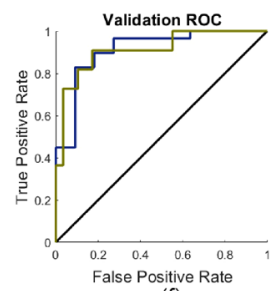

(f)

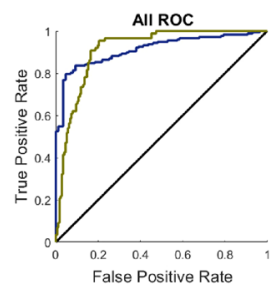

(h)

Fig. 2 Artificial neural network confusion matrix and their corresponding area under curve (AUC) for best sensitivity and 1-specificity from the network's training, validation and testing data with 10 hidden layers, 10 input elements and two outputs.

(a) Training confusion matrix; (b) validation confusion matrix; (c) test confusion matrix; (d) all confusion matrix; (e) AUC of training confusion matrix; (f) AUC of validation confusion matrix; (g) AUC of test confusion matrix; (h) AUC of all confusion matrix. ROC: receiver operating characteristic

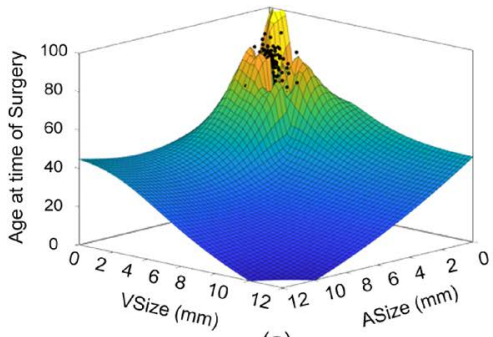

(a)

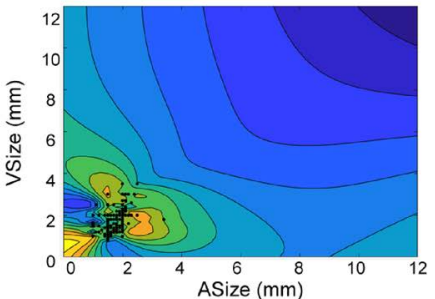

(b)

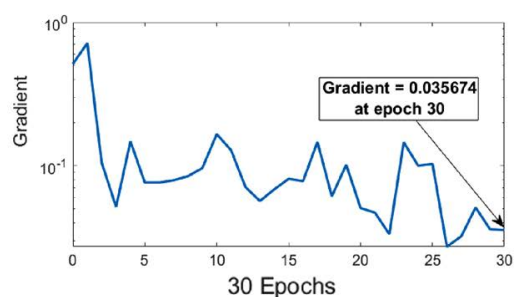

(c)

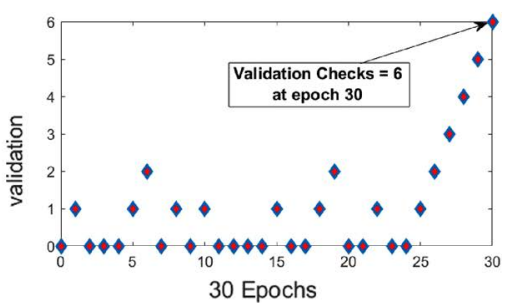

(d)

Fig. 3 (a) Three dimensional scatter plot and the cubic surface interpolant with different combinations of features (artery size and vein size vs. age at the time of surgery); (b) the 2 dimensional plot for patient's ASize (artery size) and VSize (vein size). The sample values are shown in black points; (c) the scale conjugate gradient used for training the data in artificial neural network pattern recognition with 30 epochs; (d) the epochs value used for data validation which is in the range of (0-6).

lowest level of mean squared error (MSE) at epoch 24 (range, 0-30 epoch), with the best performance validation at the gradient of 0.168989 . This is also reflected in the error autocorrelation histogram plot that correlates with itself at any given time with rapid fall of the bellshaped curve (Fig. 1). The overall accuracy of the training, validation, testing and all data on each output matrix at detection of FM was $86.4 \%, 82.5 \%, 77.5 \%$ and $84.5 \%$, respectively. The results also corresponded with the AUC for each output matrix at best sensitivity and 1-specificity with the log-rank test of probability $\mathrm{p}<0.01$ (Fig. 2). In order to evaluate the pattern recognition ability (impact of variables) of the ANN, factors of age, vein and artery size (diameter) in millimetres were subjected to similar 
assessment. This showed the gradient level of 0.035674 validated at the highest range of epoch with an accuracy of $>89 \%$, demonstrating the significance of age, vein and artery size on the outcome of FM (Fig. 3).

\section{Discussion}

The outcome of this study reveals that AI could predict FM with $>80 \%$ accuracy by only 10 given attributes (factors). This predictive model has the potential for higher sensitivity and specificity if a broader range of variables is made available for a more in-depth learning process. In contrast, an example of a higher performance on 10 variables was apparent in the study of Anagnostou et al., where AI significantly enhanced (99.38\%, $\mathrm{n}=283$ cases) the ability of early prostate cancer diagnosis with high sensitivity and specificity ( $>95 \%$ ), and inhibited a higher number of unnecessary tissue samplings with higher cancer staging capabilities (TNM staging) $(81 \%-100 \%) .{ }^{15)}$ Similar results were also noted in the early non-invasive detection of breast cancer, surgical free flap infection, survival following gastric cancer and exposure to fractures in diagnostic imaging. ${ }^{16-19)}$

In this study, the ANN classification identified three characteristics with significant influence on FM, including age, artery and vein diameter with an accuracy of $>89 \%$. The maximum aggregation points of scattered plots constructed in $2 \mathrm{D}$ and $3 \mathrm{D}$ demonstrated both vein and artery diameter $>1.8-2 \mathrm{~mm}$ to influence the FM in the positive direction (Fig. 3). This point for age was at 60 years in comparison with the mean of 63 (IQR, 22-88) calculated statistically. All factors (age, vein and artery size) were validated using ANN with the lowest gradient of error at 0.035674 at highest validation point on epoch (six being the highest, range 0-6) (Fig. 3). Such predictive examples are evident in the study of Maharlou et al. where pattern recognition by AI predicted the length of intensive care unit (ICU) stay following cardiac surgery in $n=311$ individuals with $>88 \%$ accuracy. It was noted that the two factors of the aortic clamp and the cardiopulmonary bypass duration directly influence the length of ICU stay. ${ }^{20)}$

This precision of AI owes its existence to computational analytical tools that are inspired by the biological nervous system. They are made of highly interconnected networks of computer processors known as 'neurons' or so-called 'artificial neural networks (ANNs)'. ANNs are capable of performing parallel computations for data analysis, learning from historical examples, utilising important links within a data set (that might not be apparent) and analysing non-linear data. ${ }^{21)}$ One other advantage of AI is its ability to automatically learn and continuously improve on imprecise information and to self-regulate its performance to the highest level of precision and detection. In addition, such a system is capable of including multiple prediction models at the same time with the given data set so that it can identify the best fit model. Once the best fit model is detected, this is subjected to two further independent steps of validation and testing that make AI robust and avoid statistical pitfalls or interpretations. ${ }^{22,23)}$

This is the first study in the literature that has evaluated the role of virtual AI in the prediction of FM, and its external revalidation is highly advocated. The study benefits from an adequate number (lack of comparison in the literature) of individuals with complete data and uniform type of access (RCAVF). The novel nature of the research does not create a platform for an actual and active comparison. The combination of AI and the availability of big data (quality and quantity) have the potential to produce an advanced and refined individualised evidence-based practice and permit a clinical-digital convergence. This remains dependent on the meaningful harvesting of the data and possible hidden information to full capacity that remains to be examined. The use of AI has led to a new paradigm of knowledge and information that questions old methods of data collection, study designs and randomisation that lead to medical intelligence. ${ }^{24,25)}$ However, one critical component of successful ANN depends on the creation of high quality and comprehensive data which still relies on the human factor and possible error. There is no doubt that there will be some degree of scepticism with outcomes and performance of $\mathrm{AI}$ in medicine, but their broader role in diagnostics, detection, predictive models and decisionmaking process in surgery is expanding and cannot be denied. Furthermore, a thorough understanding of ANNs and their methodology remains crucial for clinicians as unfit data could be used for incorrect decision-making in clinical practice. Overall, surgeons are in a position to integrate $\mathrm{AI}$ and modernise clinical practice for the provision of the highest quality of care, and this requires strong interdisciplinary collaboration and fostering of education for the dissemination of AI methods in medical practice.

\section{Future directions}

In this study, we only evaluated AI in the prediction of FM and of recognition of influencing variables in RCAVF. However, AI could be applied to detect FM and secondary outcomes (primary patency, primary assisted patency and secondary patency) in any type of arteriovenous fistula. As mentioned earlier AI has the potential to recognise factors (pattern recognition) that could affect any of the outcomes.

\section{Conclusion}

The application of AI in recognition of factors and prediction of FM in RCAVF has demonstrated high accuracy. AI 
is a replicable tool that could remain up to date and flexible for ongoing learning, and the role of AIs in vascular access provision remains unexplored. AI has the potential to serve as a clinical decision-making tool and further research in this upcoming filed is highly advocated.

\section{Disclosure Statement}

All authors have no conflict of interest.

\section{Author Contributions}

Study concept: AK

Data collection: AK

Writing: all authors

Critical review and revision: all authors

Final approval of the article: all authors

\section{Supplementary Information}

Supplementary information is available at the article sites on J-STAGE and PMC.

\section{References}

1) Rooijens PPGM, Tordoir JHM, Stijnen T, et al. Radiocephalic wrist arteriovenous fistula for hemodialysis: meta-analysis indicates a high primary failure rate. Eur J Vasc Endovasc Surg 2004; 28: 583-9.

2) Lok CE, Oliver MJ. Overcoming barriers to arteriovenous fistula creation and use. Semin Dial 2003; 16: 189-96.

3) Kordzadeh A, Askari A, Hoff M, et al. The impact of patient demographics, anatomy, comorbidities, and perioperative planning on the primary functional maturation of autogenous radiocephalic arteriovenous fistula. Eur J Vasc Endovasc Surg 2017; 53: 726-32.

4) Schmidli J, Widmer MK, Basile C, et al. Vascular access: 2018 clinical practice guidelines of the European Society for Vascular Surgery. Eur J Vasc Endovasc Surg 2018; 55: 757818.

5) Santangeli P, Marchlinski FE. Facts are stubborn things, but statistics are more pliable*: should we use the antimicrobial envelope for every device implant? J Cardiovasc Electrophysiol 2015; 26: 790-1.

6) Twine CP, Haidermota M, Woolgar JD, et al. A scoring system (DISTAL) for predicting failure of snuffbox arteriovenous fistulas. Eur J Vasc Endovasc Surg 2012; 44: 88-91.

7) Lok CE, Allon M, Moist L, et al. Risk equation determining unsuccessful cannulation events and failure to maturation in arteriovenous fistulas (REDUCE Ftm I). J Am Soc Nephrol 2006; 17: 3204-12.

8) Hamet P, Tremblay J. Artificial intelligence in medicine. Metabolism 2017; 69: S36-40.

9) Theofilatos K, Pavlopoulou N, Papasavvas C, et al. Predicting protein complexes from weighted protein-protein interaction graphs with a novel unsupervised methodology: evolutionary enhanced Markov clustering. Artif Intell Med
2015; 63: 181-9.

10) Banerjee S. Fistula maturation and patency for successful dialysis. Dial Transplant 2009; 38: 442.

11) World Health Organization. Global Status Report on noncommunicable diseases: attaining the nine global non-communicable diseases targets; a shared responsibility. Geneva: World Health Organization, 2014.

12) Fihn SD, Blankenship JC, Alexander KP, et al. ACC/AHA/ AATS/PCNA/SCAI/STS Focused update of the guideline for the diagnosis and management of patients with stable ischemic heart disease. A report of the American College of Cardiology/American Heart Association Task Force on Practice Guidelines, and the American Association for Thoracic Surgery, Preventive Cardiovascular Nurses Association, Society for Cardiovascular Angiography and Interventions, and Society of Thoracic Surgeons. Circulation 2014; 130: 1749-67.

13) Walsh P, Cunningham $P$, Rothenberg $S$, et al. An artificial neural network ensemble to predict disposition and length of stay in children presenting with bronchiolitis. Eur J Emerg Med 2004; 11: 259-64.

14) Khan J, Wei JS, Ringner M, et al. Classification and diagnostic prediction of cancers using gene expression profiling and artificial neural networks. Nat Med 2001; 7: 673-9.

15) Anagnostou T, Remzi M, Lykourinas M, et al. Artificial neural networks for decision-making in urologic oncology. Eur Urol 2003; 43: 596-603.

16) Herman-Saffar O, Boger Z, Libson S, et al. Early noninvasive detection of breast cancer using exhaled breath and urine analysis. Comput Biol Med 2018; 96: 227-32.

17) Kuo PJ, Wu SC, Chien PC, et al. Artificial neural network approach to predict surgical site infection after free-flap reconstruction in patients receiving surgery for head and neck cancer. Oncotarget 2018; 9: 13768-82.

18) Oh SE, Seo SW, Choi MG, et al. Prediction of overall survival and novel classification of patients with gastric cancer using the survival recurrent network. Ann Surg Oncol 2018; 25: 1153-9.

19) Chung SW, Han SS, Lee JW, et al. Automated detection and classification of the proximal humerus fracture by using deep learning algorithm. Acta Orthop 2018; 89: 468-73.

20) Maharlou H, Niakan Kalhori SR, Shahbazi S, et al. Predicting length of stay in intensive care units after cardiac surgery: comparison of artificial neural networks and adaptive neuro-fuzzy system. Healthc Inform Res 2018; 24: 10917.

21) Ramesh AN, Kambhampati C, Monson JRT, et al. Artificial intelligence in medicine. Ann R Coll Surg Engl 2004; 86: 334-8.

22) Steimann F. On the use and usefulness of fuzzy sets in medical AI. Artif Intell Med 2001; 21: 131-7.

23) Hashimoto DA, Rosman G, Rus D, et al. Artificial intelligence in surgery: promises and perils. Ann Surg 2018; 268: 70-6.

24) Patel VL, Shortliffe EH, Stefanelli M, et al. The coming of age of artificial intelligence in medicine. Artif Intell Med 2009; 46: 5-17.

25) Zupan B, Holmes JH, Bellazzi R. Knowledge-based data analysis and interpretation. Artif Intell Med 2006; 37: 1635 . 